www.jmscr.igmpublication.org

Index Copernicus Value: 79.54

ISSN (e)-2347-176x ISSN (p) 2455-0450

crossrefDOI: https://dx.doi.org/10.18535/jmscr/v7i3.145

\title{
Adenomyoepithelioma of the Breast in the young- A Rare Case
}

\section{Authors \\ Dr Sreeramulu P N ${ }^{1}$, Dr Tejaswini M Pawar ${ }^{2}$, Dr Srinivasan $D^{3}$, Dr Spurthi $\mathrm{S}^{4}$, Dr Swaroop Raj B V}

Department of General Surgery, RL Jalappa Hospital, Tamaka, Kolar

\begin{abstract}
Myoepithelioma/adenomyoepithelioma is a rare salivary gland tumor arising from proliferation of myoepithelial cells. The presence of this tumor is rare in the breast. In this case report we encounter adenomyoepithelioma of the breast in a young female. The incidence is rare in younger individuals.

Keywords: breast, adenomyoepithelioma.
\end{abstract}

\section{Introduction}

Myoepithelioma/adenomyoepithelioma is a rare salivary gland tumor arising from proliferation of myoepithelial cells. These tumors represent $1 \%-1.5 \%$ of all salivary gland tumors, and are distributed $48 \%$ in parotids, $42 \%$ in the small salivary glands and the remaining in glandula submandibularis and seromucous glands of the nose and larynx. ${ }^{[1]}$ Other localizations reported are the skin, breast, chest, lung and pancreas. ${ }^{[1]}$ Incidence of myoepithelioma in breast could not be ascertained due rarity of the lesion and paucity of literature on the subject with few case reports.

\section{Case Report}

A 16 year old girl came to the surgery OPD of $R$ L Jalappa hospital and research centre with complaints of lump in her left breast- inner upper and outer quadrants which she noticed 3 months ago which was initially small in size and later progressed to the present size. The skin around the swelling was normal and there was no nipple discharge. The patient gave no history of any trauma. Considering the age of the patient and the presentation of the lump, giant fibroadenoma of the breast was taken as the likely diagnosis. Patient was advised for FNAC and USG of the breast preoperatively, which showed features consistent with fibroadenoma. Excision of the lump was planned and performed and the specimen was sent for Histopathological examination. Excised specimen was a well circumscribed, solitary, white firm nodule measuring about $10 \mathrm{~cm} \times 6 \mathrm{~cm}$ in dimension well encapsulated. Histopathology showed features suggestive of Adenomyoepithelioma of left breast. 


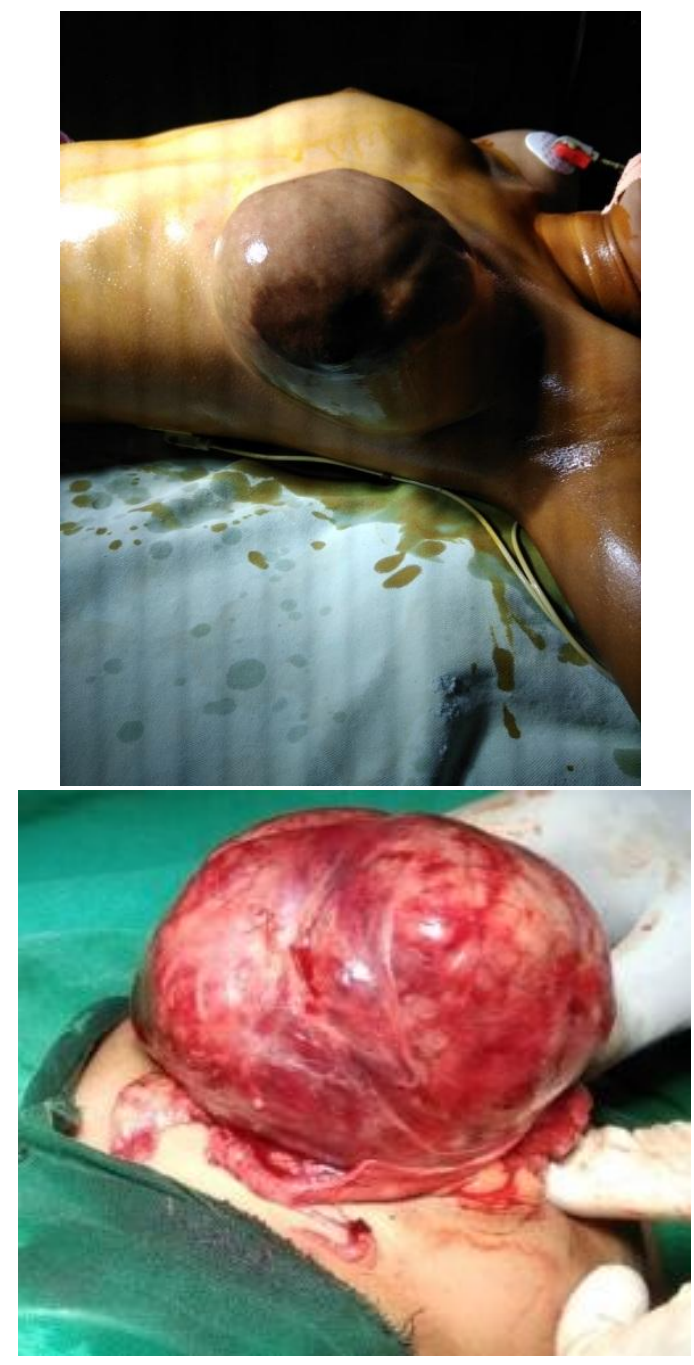

Figure 1 Left breast - swelling preoperative and swelling intra operative

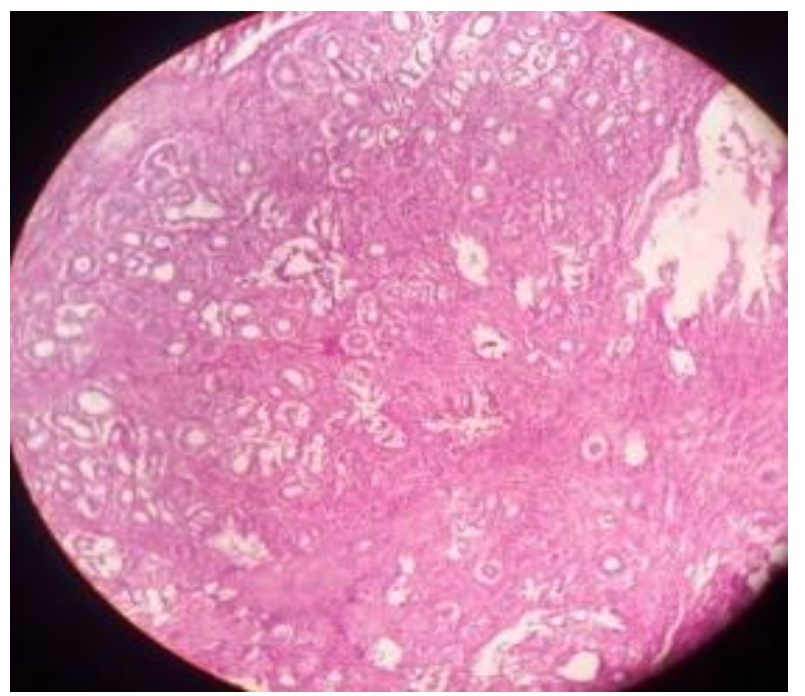

Figure 2: Microscopy shows a well delineated tumour with lobules showing tubular patternadenomyoepithelioma

\section{Discussion}

Adenomyoepithelioma (AME) first described by Hamperl in the year 1970, which showed that most tumours are benign, but malignant degeneration, although very unusual, may occur. Adenomyoepithelioma's have been classified as tubular, lobulated, or spindle variants, on the basis of their growth pattern. Malignant neoplasms have been described in many patterns and have been sub-classified as: undifferentiated, myoepithelial, or epithelial. Metastasis is only documented in tumours that are $2 \mathrm{~cm}$ or larger, and appear to be hematogenous rather than lymphatic ${ }^{2}$.

Tumors with bicellular proliferation of both epithelial and myoepithelial cells are called adenomyoepitheliomas $^{3}$. The adenomyoepithelioma is a biphasic tumor, either benign or with low potential of malignancy, which can be found in salivary gland, skin adnexal and lung, but is not frequent in the mammary gland ${ }^{4}$. Differential diagnosis of adenomyoepithelioma includes sclerosing adenosis, fibroadenoma, tubular adenoma, and pleomorphic adenoma ${ }^{[3]}$.

Tavassoli proposed a classification system of myoepithelial lesions of the breast, sub-classifying into: myoepitheliosis, Adenomyoepithelioma and malignant myoepithelioma. The same author classified AME as spindle cell, lobulated, and tubular (or adenosis) types with carcinoma arising in $\mathrm{AME}^{1}$. Three variants of AMEs were described by Tavassoli.

The first variant is the tubular pattern, which is characterized by a balanced proliferation of rounded tubules, as well as unusually prominent and hyperplastic myoepithelial cells.

The second variant is the spindle cell type, which is composed of a predominantly spindled myoepithelial cell proliferation admixed with a few columnar, epithelial-lined tubules.

The third variant exhibits a lobular pattern composed of solid nests of myoepithelial cells proliferating around compressed tubules; the solid nests of tumor are then surrounded by fibrous connective tissue septa of varying thicknesses ${ }^{5}$. 
WHO Classification of myoepithelial lesion of the breast

1. Myoepitheliosis
a) Intraductal
b) Periductal

2. Adenomyoepithelial adenosis

3. Adenomyoepithelioma

a. Benign

b. With malignant changes (specify the subtype)

- Myoepithelial carcinoma arising in an adenomyoepithelioma

- Epithelial carcinoma arising in an adenomyoepithelioma

- Malignant epithelial and myoepithelial components

- Sarcoma arising in adenomyoepithelioma

- Carcinosarcoma arising in adenomyoepithelioma

4. Malignant myoepithelioma (myoepithelial (arcinoma) ${ }^{2}$.

Adenomyoepitheliomas may vary in size from 1 $\mathrm{cm}$ to $21 \mathrm{~cm}$, the average being $2.5 \mathrm{~cm}$. Because of the varied and hypercellular nature of these tumors the diagnosis by cytology is difficult and core biopsy may be more accurate. ${ }^{1}$ Histologically the recognition of the biphasic cellular elements and the characteristic overall architecture of the tumors in combination with immunohistochemistry are essential to establish the correct diagnosis.

In the above case the initialy Fine needle aspiration cytology showed features of fibroadenoma which later on histopathology turned out to be adenomyoepithelioma.

The myoepithelial cells express typical cytokeratins of the basal layer of stratified epithelia (CK5, CK14, and CK17), filamentous $\alpha$ smooth muscle actin (SMA) and the heavy chainmyosin (hc-myosin). Some tumor suppressor proteins which include -p63, p73, 14-3-3 sigma, maspin and Wilms Tumor (WT-1) have been preferentially detected in myoepithelial cells. Myoepithelial cells rarely transform; however, when they do transform, they generally give rise to benign or low-grade malignant tumors. The lesions which show a myoepithelial component are rare in the mammary gland, and they are often benign and biphasic, with an epithelial and a myoepithelial basal component ${ }^{4}$.

The presence of regularly spaced, rounded or ovoid glands; unidirectional streaming of the glands; and prominent clear cell or spindle cell myoepithelium are some morphologic clues to the diagnosis of $\mathrm{AME}^{4}$. Differential diagnosis of adenomyoepithelioma includes sclerosing adenosis, fibroadenoma, tubular adenoma, and pleomorphic adenoma ${ }^{2}$. When the tumor predominantly displays a spindle cell component, it may morphologically be mistaken for a myoid hamartoma or leiomyoma. Strong reactivity for S100 and p63, and minimal staining for actin and cytokeratin in AME are helpful in differentiating the 2 lesions. Immunostains for myoepithelial markers, especially p63, are useful for highlighting the abundant myoepithelial components. $^{5}$

Majority of the cases of AME are reported in the fifth and sixth decade of life. It is uncommon in young patients but is occasionally seen in the third decade. Our patient was a very young female of 16 years. Rare cases of AME are seen in men ${ }^{5}$.Prognosis of patients with benign AME of the breast is usually good, but it has a potential for local recurrence, especially in the tubular and lobulated variants. Total surgical excision with an adequate margin of uninvolved breast tissue is therefore recommended ${ }^{2}$.

\section{Conclusion}

Adenomyoepithelioma of left breast in a very young girl of 16 years, which is most common in fifth and sixth decade, very few cases are reported. diagnosis by cytology is difficult and core biopsy may be more accurate. Recognition of the biphasic cellular elements and the characteristic overall architecture of the tumors histologically in combination with immunohistochemistry are essential to establish the correct diagnosis. 


\section{References}

1. Khan L, Srivastava S, Singh PK, Ather M. Benign breast myoepithelioma. Journal of Cytology. January 2013. Volume 30. Issue 1.

2. Ahmad SS, Hassan M, Alam S, Aftab F. A clinical dilemma: Malignant adenomyoepithelioma of the breast - case report and review of literature. Case studies in surgery. 2015; 2(1); 64- 68.

3. V Satyanarayana, S Gole. Adenomyoepithelioma A Rare Breast Tumor: Case Studies With Review Of The Literature; Case Studies With Review of The Literature. The Internet Journal of Pathology. 2012 Volume 13 Number 2.

4. Vincenzo Petrozza et al. Breast adenomyoepithelioma: a case report with malignant proliferation of epithelial and myoepithelial elements. World Journal of Surgical Oncology 2013, 11:285

5. Ji Yoon Yoon, Dhananjay Chitale, Adenomyoepithelioma of the Breast. A Brief Diagnostic Review. Arch Pathol Lab Med-Vol 137, May 2013. 725-729.

6. Pradhan S, Yadav RP. Adenomyoepithelioma of breast- Case Report. Journal of Pathology of Nepal (2011) Vol. 1, 154 -157. 